\title{
High-resolution, label-free two-photon imaging of diseased human corneas
}

\author{
Ana Batista \\ Hans Georg Breunig \\ Aisada König \\ Andreas Schindele \\ Tobias Hager \\ Berthold Seitz \\ Karsten König
}




\title{
High-resolution, label-free two-photon imaging of diseased human corneas
}

\author{
Ana Batista, ${ }^{a, b}$ Hans Georg Breunig, ${ }^{a, b}$ Aisada König, ${ }^{a, b}$ Andreas Schindele, ${ }^{b}$ Tobias Hager, ${ }^{c, d}$ Berthold Seitz, ${ }^{c, d}$ \\ and Karsten König ${ }^{\mathrm{a}, \mathrm{b}, *}$ \\ a Saarland University, Department of Biophotonics and Laser Technology, Saarbrücken, Germany \\ bJenLab GmbH, Jena, Germany \\ 'Saarland University, Department of Ophthalmology, Medical Center, Homburg/Saar, Germany \\ 'Saarland University, Lions Cornea Bank Saar-Lor-Lux, Trier/Westpfalz, Medical Center, Homburg/Saar, Germany
}

\begin{abstract}
The diagnosis of corneal diseases may be improved by monitoring the metabolism of cells and the structural organization of the stroma using two-photon imaging (TPI). We used TPI to assess the differences between nonpathological (NP) human corneas and corneas diagnosed with either keratoconus, Acanthamoeba keratitis, or stromal corneal scars. Images were acquired using a custom-built five-dimensional laser-scanning microscope with a broadband sub-15 femtosecond near-infrared pulsed excitation laser and a 16-channel photomultiplier tube detector in combination with a time-correlated single photon counting module. Morphological alterations of epithelial cells were observed for all pathologies. Moreover, diseased corneas showed alterations to the cells' metabolism that were revealed using the $\mathrm{NAD}(\mathrm{P}) \mathrm{H}$ free to protein-bound ratios. The mean autofluorescence lifetime of the stroma and the organization of the collagen fibers were also significantly altered due to the pathologies. We demonstrate that TPI can be used to distinguish between NP and diseased human corneas, based not only on alterations of the cells' morphology, which can also be evaluated using current clinical devices, but on additional morphological and functional features such as the organization of the stroma and the cells' metabolism. Therefore, TPI could become an efficient tool for diagnosing corneal diseases and better understanding the biological processes of the diseases. ๑ 2018 Society of Photo-Optical Instrumentation Engineers (SPIE) [DOI: 10.1117/1.JBO.23.3.036002]
\end{abstract}

Keywords: corneal imaging; corneal disease; corneal diagnosis; fluorescence lifetime imaging; second-harmonic generation; twophoton microscopy.

Paper 170752PR received Nov. 20, 2017; accepted for publication Feb. 8, 2018; published online Mar. 2, 2018.

\section{Introduction}

Corneal dysfunctions are the second major cause of blindness worldwide, surpassed only by cataracts. ${ }^{1}$ Precise and early diagnoses to enable adequate treatments of corneal pathologies are essential to reduce visual impairment. Still widely used are imaging techniques, such as the slit lamp microscope, developed over a century ago, to evaluate the anterior segment of the eye. ${ }^{2}$ The rather limited resolution with no cellular information has been surpassed by specular ${ }^{3}$ and confocal microscopy. ${ }^{4}$ Specular microscopy visualizes the morphologies of healthy and disease-affected endothelial cells, ${ }^{5}$ whereas confocal microscopy opens the access to the morphology of cells from all cornea layers and nerve densities. It is widely used in clinical practice to diagnose cornea diseases and to monitor tissue recovery following surgery. ${ }^{6}$ In combination with further corneal diagnostic methods like anterior segment optical coherence tomography and corneal topography, these techniques have greatly improved corneal diagnosis. However, they fail to provide information on the cell metabolism and disregard the morphological organization of the major layer of the cornea-the stroma.

A corneal clinical imaging modality based on two-photon imaging (TPI) could overcome these limitations and provide information based on earlier detectable signs of pathology,

*Address all correspondence to: Karsten König, E-mail: k.koenig@blt. uni-saarland.de thereby further improving the diagnostic of corneal pathologies. TPI can be used to evaluate the metabolic state of cells by fluorescence lifetime imaging microscopy (FLIM). ${ }^{7}$ With FLIM, the free and protein-bound components of the metabolic cofactors can be quantified, and thereby the oxidation-reduction ratio and consequently the metabolic activity of cells can be indirectly determined. ${ }^{8}$ Furthermore, based on second-harmonic generation (SHG) generated by structures with noncentrosymmetric molecular organization, ${ }^{9}$ the corneal stroma collagen fibers can be visualized, and alterations induced by pathologies to the stroma microstructure can be assessed. The feasibility of TPI to image and characterize the cornea has been previously demonstrated ex vivo ${ }^{10-15}$ and in the cornea of rabbits in vivo. ${ }^{16}$

In this proof of principle study, we demonstrate the feasibility of using TPI to distinguish between nonpathological (NP) and diseased corneas, based on cell morphology, cell metabolism, and the stromal autofluorescence (AF) lifetime and fibers organization. For that purpose, TPI was used to evaluate differences between NP corneas and samples affected by keratoconus (KC), a disorder that affects the corneal shape, characterized by a progressive corneal thinning, stepping, and formation of a cone. ${ }^{5,17}$ Individual samples affected by Acanthamoeba keratitis (AK), a corneal pathology caused by an amoeba that causes ulceration and necrosis of the epithelium and the stroma, ${ }^{5,18,19}$ and stromal corneal scars (SCS), which can be

$1083-3668 / 2018 / \$ 25.00$ @ 2018 SPIE 
formed during atypical wound healing following cornea injury, infection, or refractive surgery, ${ }^{20}$ were also evaluated.

The cells' AF intensity images revealed alterations of the corneal epithelial cell morphology, whereas the AF lifetime imaging of the metabolic cofactor nicotinamide adenine dinucleotide (NADH)/nicotinamide adenine dinucleotide phosphate (NADPH) - NAD(P)H was used to assess changes to the cells' metabolism. Alterations of the corneal stroma structural organization as well as to the AF lifetime were also investigated. To the best of our knowledge, this is the first time that TPI of the corneal epithelium and stroma is jointly used to characterize these pathologies. We demonstrate that TPI can provide information on the cornea that is inaccessible by current clinical devices. This information could be used for the diagnostic of corneal diseases, as well as for a better understanding of their mechanisms of action.

\section{Methods}

\subsection{Sample Preparation}

Human corneal buttons were obtained from the Lions Cornea Bank Saar-Lor-Lux, Trier/Westpfalz at the Department of Ophthalmology, Saarland University Medical Center, Homburg/ Saar, Germany. The samples were excluded from corneal transplantation due to low endothelial cell counts (EDC). The samples had on average an EDC of $(1808 \pm 16)$ cell $/ \mathrm{mm}^{2}$. However, the corneas originated from healthy donors and yielded negative results in serology and microbiology tests. These samples were classified as NP, and a total of four samples with storage times up to 1 week were imaged. Prior to image acquisition, the samples were stored in Culture Medium II with Dextran T500 (\#F9017, Biochrom GmbH, Berlin, Germany) supplemented with 5\% new-born calf serum (\#S0415, Biochrom $\mathrm{GmbH}$, Berlin, Germany) at $37^{\circ} \mathrm{C}$ under a $5 \% \mathrm{CO}_{2}$ atmosphere. ${ }^{21}$

Pathological corneas of patients suffering from $\mathrm{KC}(n=5)$, $\mathrm{AK}(n=1)$, and $\operatorname{SCS}(n=1)$ were obtained from the
Department of Ophthalmology, University of Saarland, Medical Center, Homburg/Saar, Germany. After surgery, the samples were stored using the same conditions as the NP corneal buttons to minimize time-induced alterations to the metabolic activity of the cells. The samples were collected and imaged within $24 \mathrm{~h}$ after keratoplasty.

During image acquisition, all corneal samples were kept hydrated using a live cell imaging solution (\#A14291DJ, Life Technologies). All procedures were conducted following the World Medical Association's Declaration of Helsinki for scientific use of human corneas. All patients gave written informed consent for the use of their tissue for scientific research.

\subsection{Two-Photon Microscope}

A near-infrared (NIR) femtosecond (fs) five-dimensional (5-D) multiphoton laser-scanning microscope with axial, lateral, temporal, and spectral resolutions of (1 to 2) $\mu \mathrm{m}, 300 \mathrm{~nm}, 100 \mathrm{ps}$, and $12.5 \mathrm{~nm}$, respectively, was used for image acquisition. The system (Fig. 1) consisted of an inverted microscope with a galvanometric scanner unit, a beam expander, a dichroic mirror, and a $40 \times$ NA 1.3 oil immersion objective for laser light focusing and signal collection in reflection geometry. Sample excitation was accomplished using a mode-locked titanium-sapphire laser (Integral Pro 400; FemtoLasers Produktions $\mathrm{GmbH}$, Vienna, Austria) generating $\sim 10$-fs pulses at a frequency of $85 \mathrm{MHz}$. The laser emission with an M-shaped spectral profile was centered at $\sim 800 \mathrm{~nm}$, i.e., it contained two main peaks at 775 and $830 \mathrm{~nm}$ and had a full width at half maximum (FWHM) of about $95 \mathrm{~nm}$ (Fig. 1), allowing the simultaneous excitation of multiple endogenous fluorophores. The group velocity dispersion of the optics was precompensated using chirped mirrors so that laser pulse durations were below $15 \mathrm{fs}$ behind the microscope objective. Up to 13 images with $256 \times 256$ pixels with a scanning speed of $7.4 \mathrm{~s}$ per frame were summed to collect enough photons for data analysis. The corneas were imaged using mean laser powers between 10 and $15 \mathrm{~mW}$.

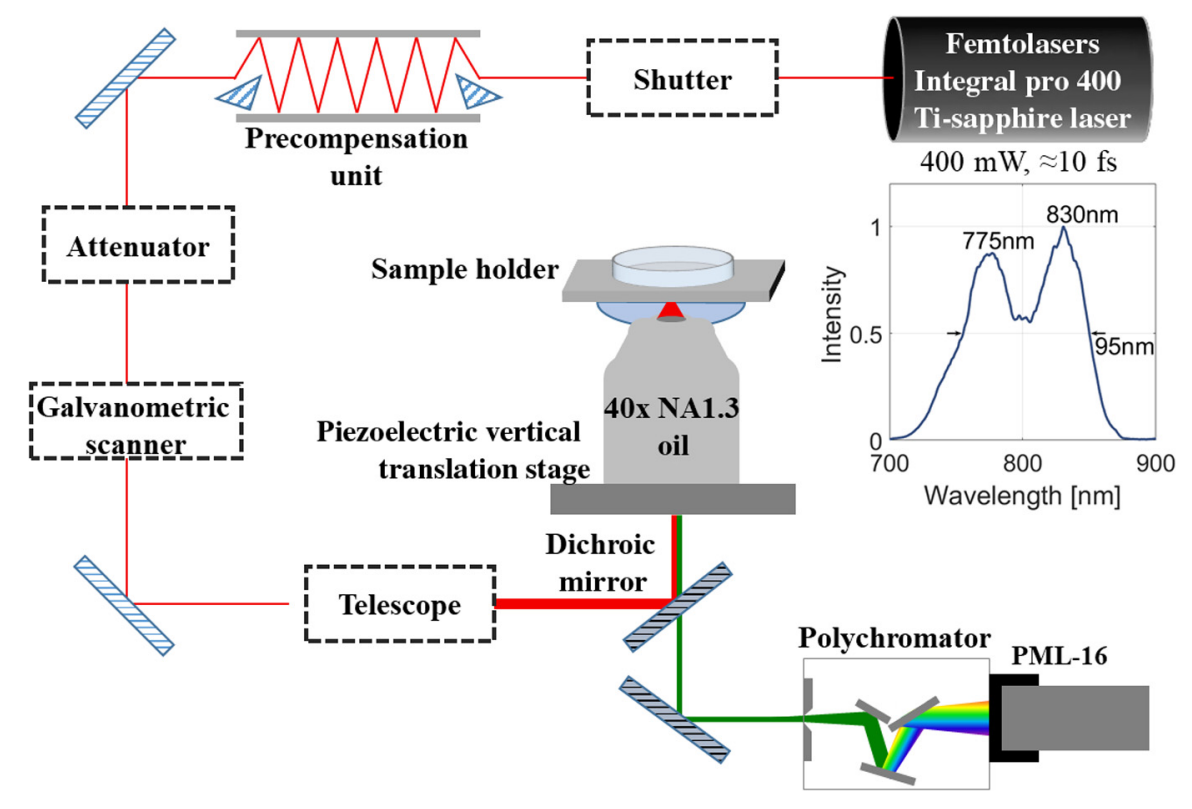

Fig. 1 Schematic setup of the 5-D laser scanning microscope used for TPI of the cornea. The system has axial, lateral (three-dimensional), temporal (four-dimensional), and spectral (5-D) resolutions of (1 to 2 ) $\mu \mathrm{m}, 300 \mathrm{~nm}, 170 \mathrm{ps}$, and $12.5 \mathrm{~nm}$, respectively. The laser spectrum is shown. The pulse width at the sample was below $15 \mathrm{fs}$. 
$\mathrm{AF}$ and SHG photons were detected between 400 and $600 \mathrm{~nm}$ using a 16 channels photomultiplier tube (PMT) detector with 12.5-nm spectral resolution (16PML-PMT, Hamamatsu, Japan) associated with a time-correlated single photon counting SPC-150 module (Becker \& Hickl GmbH, Berlin, Germany).

\subsection{Image Analysis}

The assignment of the signals to individual fluorophores was accomplished by spectrally separated detection. The spectral ranges of analysis were determined based on the spectra of the tissue layers as well as the spectra of pure endogenous fluorophores. Spectrally resolved analysis of the corneal epithelial cells revealed $\mathrm{NAD}(\mathrm{P}) \mathrm{H}$ AF. The contribution of other fluorophores including flavins was negligible (data not shown). As such, epithelial cells' AF signals were analyzed between 425 and $500 \mathrm{~nm}$ and were assigned to NAD(P)H AF from mitochondria and cytoplasm. ${ }^{22}$ The spectra of the cornea stroma showed the contribution of collagen SHG and AF as well as keratocytes NAD(P)H AF (data not shown). Thus, ranges between 400 and $425 \mathrm{~nm}$ and between 425 and $575 \mathrm{~nm}$ were used to analyze the SHG and AF signals, respectively. ${ }^{22}$

Fluorescence lifetimes were determined using the commercial software SPCImage (Becker \& Hickl GmbH, Berlin, Germany) by fitting the decay data with an exponential function in the form $F(t)=\sum_{i=1}^{n} a_{i} e^{-t / \tau_{i}}$, where $F(t)$ represents the fluorescence intensity at time $t$, and $a_{i}$ are the fractional contributions of the fluorescence lifetimes $\tau_{i}$. Up to three nonoverlapping regions were imaged and analyzed for each sample.

The orientation of the stroma-collagen fibers was analyzed in equally sized images with $400 \times 400$ pixels corresponding to an area of $80 \times 80 \mu \mathrm{m}^{2}$ using a fast Fourier transform (FFT) method adapted from Mega et al. ${ }^{23}$ The method consists of applying a bandpass filter to the 2D-FFT of an image to reduce very low and high spatial frequencies. Then, a shifting histogram and a median filter were applied to adjust contrast and to smooth the FFT-images, respectively. The frequency of the different orientations was obtained using the radon transform. The degree of organization of the stroma fibers was assessed by retrieving the main orientation and the peak prominence (PP) of the main orientation defined by $\mathrm{PP}=\sqrt{\frac{1}{n-1} \sum_{o \in O}[f(d)-f(o)]^{2}}$, where $O$ is a finite set of orientations, $n$ is the cardinality of $O, f(o)$ is the frequency of the orientation $o$ derived by the radon transform, and $d=\operatorname{argmax}_{o \in O} f(o)$ is the dominant orientation. PP correlates to the PP of the frequency function $f(o)$. A high PP value indicates that $f(o)$ has a sharp peak at the dominant orientation and as such the collagen fibers are well organized, while a low PP value indicates that $f(o)$ has a disperse peak and that the collagen fibers are not well organized along the dominant orientation.

\subsection{Statistical Analysis}

Statistical analysis of the data was performed with GraphPad Prism 6 (GraphPad Software, Inc., California). The data failed in the normal distribution test. Therefore, the analysis was accomplished using the nonparametric test of Mann-Whitney U. $P$ values below 0.05 were considered significant. For AK and SCS, statistical analysis was not performed due to the sparse number of samples in each condition.



Fig. 2 AF intensity images of the epithelial layers of NP human corneas and corneas with KC, AK, and SCS. The different cell types of the epithelium (superficial, wing, and basal cells) and their respective depths are shown for each condition. In AK, red arrows point to regions where basal cells are absent. In SCS, white arrows point to autofluorescent deposits. Scale bar $=30 \mu \mathrm{m}$. 

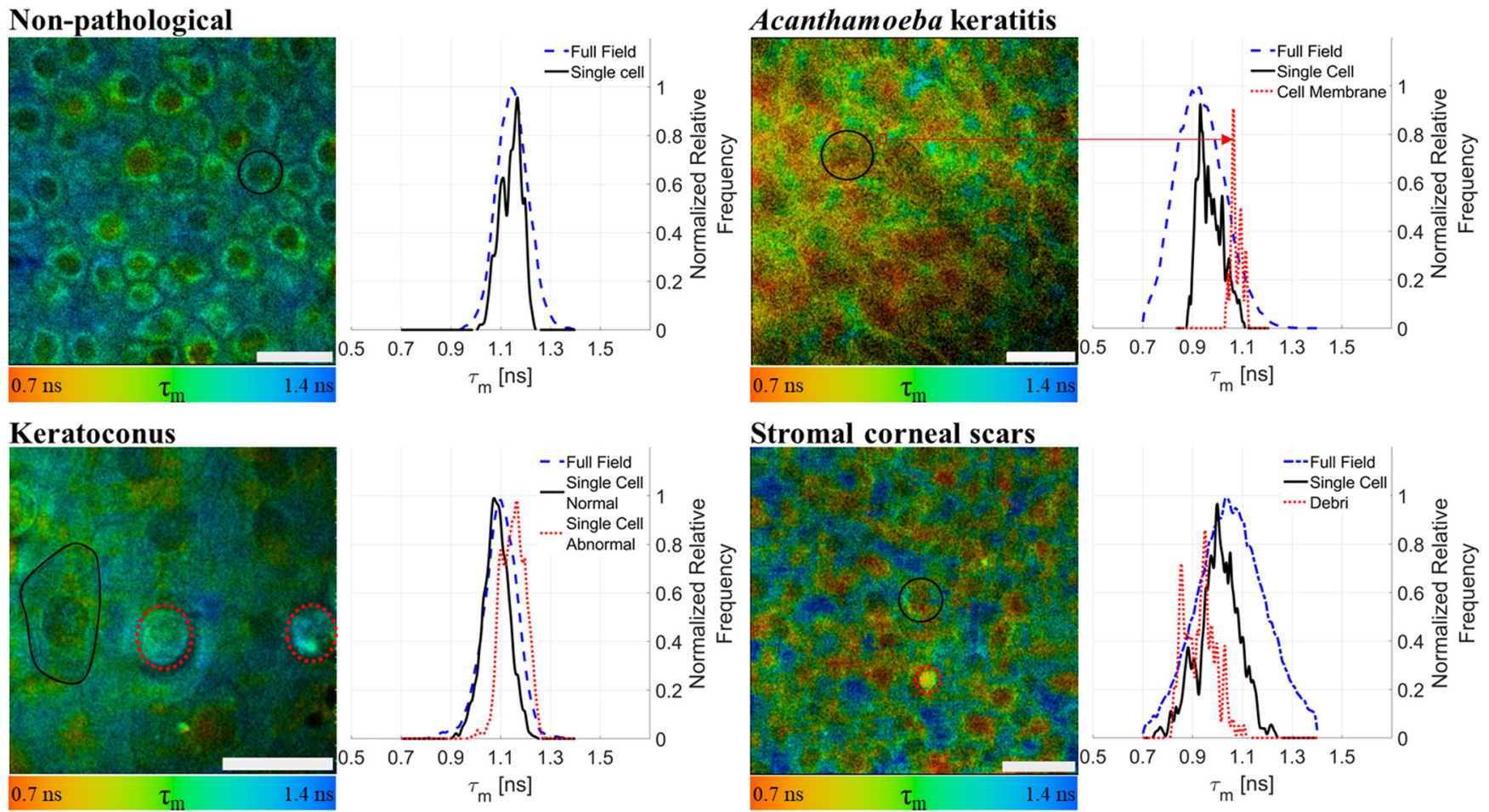

Fig. $3 \mathrm{AF}$ lifetime images and corresponding lifetime distributions of nonpathological (NP) corneas, and corneas diagnosed with keratoconus (KC), Acanthamoeba keratitis (AK), and stromal corneal scars (SCS). For all conditions, the lifetime distributions of the entire field-of-view and regions of interest (ROIs) including single cells are shown in dashed line (blue) and in full line (black), respectively. For $\mathrm{KC}, \mathrm{AK}$, and SCS corneas, the lifetime distribution of ROls of cells with altered/abnormal morphology, the cellular membrane, and debris, respectively, is additionally shown in dotted line (red). The ROI boundaries are indicated in the lifetime images with black and yellow lines. Scale bar $=30 \mu \mathrm{m}$.

\section{Results}

\subsection{Pathology-Induced Changes of the Corneal Epithelium}

Pathology-induced morphological alterations to epithelial cells of different types (superficial, wing, and basal cells) were observed in AF images (Fig. 2). In pathological samples, the cells are irregularly shaped within each layer. Furthermore, an enlargement of the basal cells was observed for $\mathrm{KC}$ and SCS corneas when compared with NP samples. In KC corneas, an elongation of the cells was also observed (Fig. 2). AF from the cell membranes was detected for the AK cornea. Moreover, regions without corneal basal cells appear (Fig. 2, red arrows). In the SCS cornea, bright autofluorescent spots, possibly related to debris deposition, are visible throughout the epithelium (Fig. 2, white arrows). A decrease in the epithelial thickness was observed for all pathological conditions. The epithelial thickness decreased from approximately $(48 \pm 6) \mu \mathrm{m}$ in NP corneas to $(31 \pm 9) \mu \mathrm{m},(25 \pm 5) \mu \mathrm{m}$, and $(30 \pm 4) \mu \mathrm{m}$ in $\mathrm{KC}, \mathrm{AK}$, and SCS, respectively.

Alterations induced by the pathologies can be highlighted in the AF lifetime images and their corresponding lifetime distributions (Fig. 3). The epithelial cells in NP corneas have a homogenous AF lifetime distribution, i.e., all cells in the field-of-view have similar $\tau_{m}$ values, whereas in $\mathrm{KC}, \mathrm{AK}$, and SCS corneas heterogenous AF lifetime distributions can be seen (Fig. 3). Cells with altered/abnormal morphology in $\mathrm{KC}$ have a longer $\tau_{m}$ than the surrounding cells with normal morphology. In the $\mathrm{AK}$ cornea, the membrane $\mathrm{AF}$, which is absent in NP and the other pathological conditions, has a longer mean lifetime than the AF of the cytoplasm of the same cells. In the SCS cornea, the bright autofluorescent spots (debris) have a homogenous and short lifetime (Fig. 3).

The NAD(P)H AF lifetime was used to assess alterations induced by the pathologies to the epithelial cell metabolism (Fig. 4). KC corneas have significantly higher ratios between free $\left(a_{1}\right)$ and protein-bound $\left(a_{2}\right)$ components of NAD(P)H $\left(a_{1} / a_{2}\right.$ ratio) than the NP corneas (Fig. 4). For AK and SCS corneas, a tendency for an increase in the metabolic cofactor $a_{1} / a_{2}$ ratio is also observed (Fig. 4).

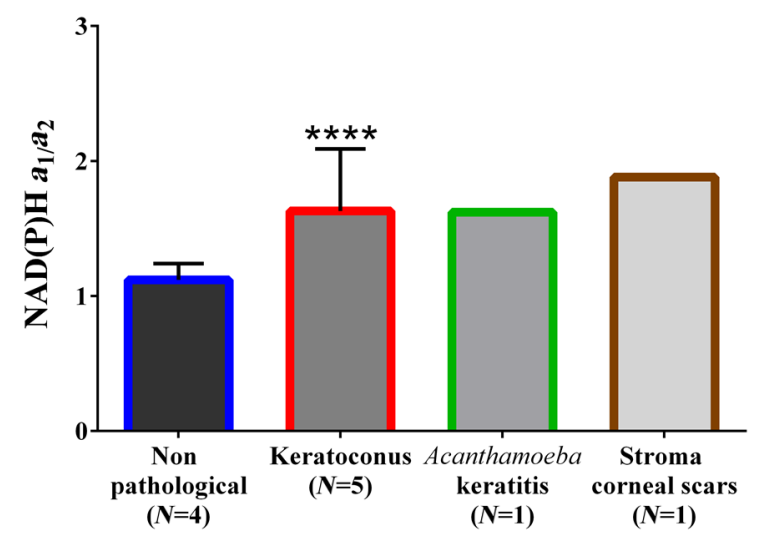

Fig. 4 Epithelial cell $\mathrm{NAD}(\mathrm{P}) \mathrm{H}$ free to protein-bound $\left(a_{1} / a_{2}\right)$ ratios of nonpathological and diseased corneas. Values represent mean \pm standard deviation. ${ }^{* \star \star}$ Statistically significant at $p<0.0001$. 


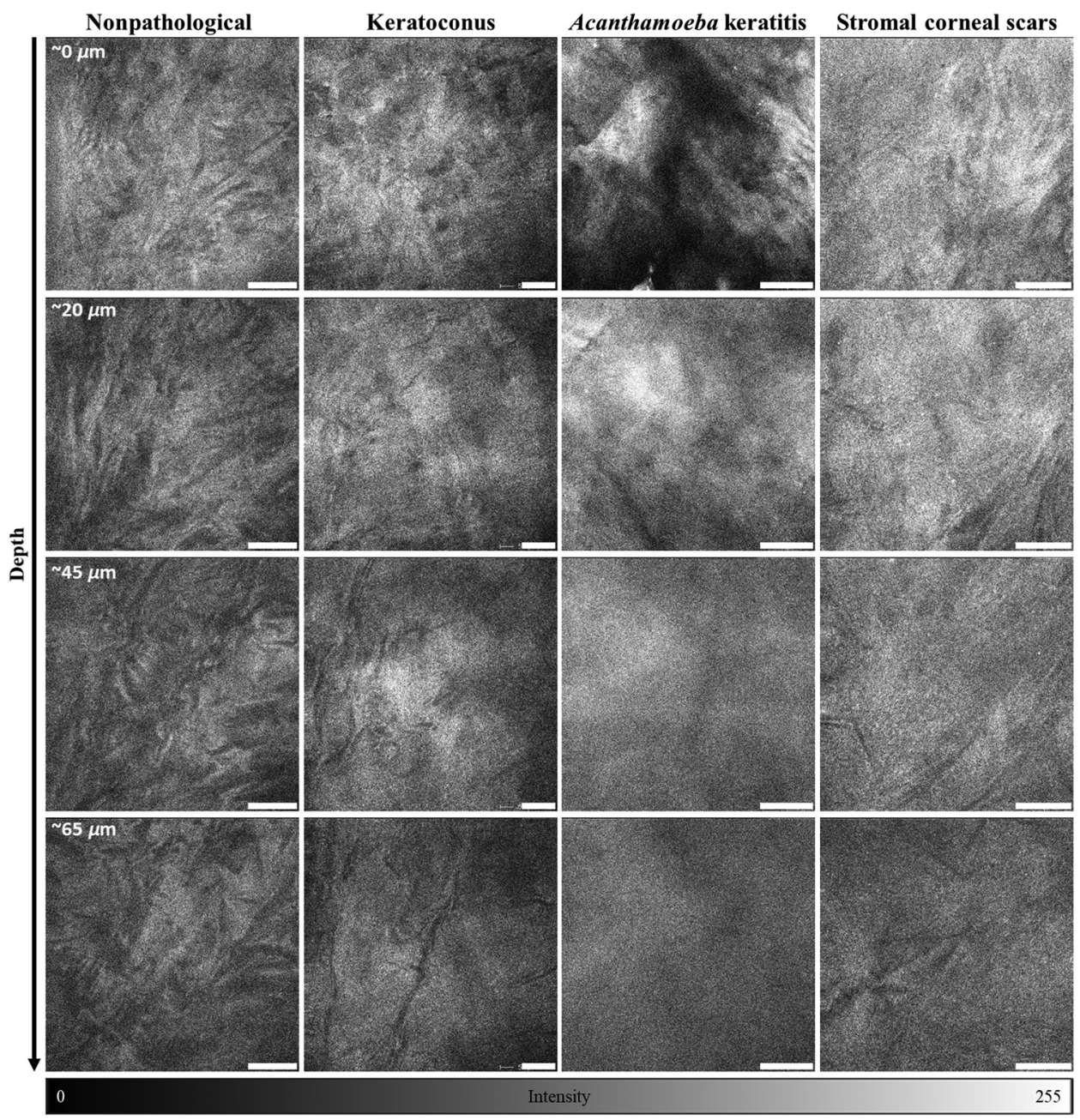

Fig. 5 SHG images of nonpathological human corneas and corneas with AK, KC, and SCS at depths of $0,20,45$, and $5 \mu \mathrm{m}$. Scale bar $=30 \mu \mathrm{m}$.

\subsection{Pathology-Induced Changes to the Cornea Stroma}

SHG images were used to assess morphological alterations induced by pathologies to the stroma, the main layer of the human cornea. Figure 5 shows SHG images of the anterior

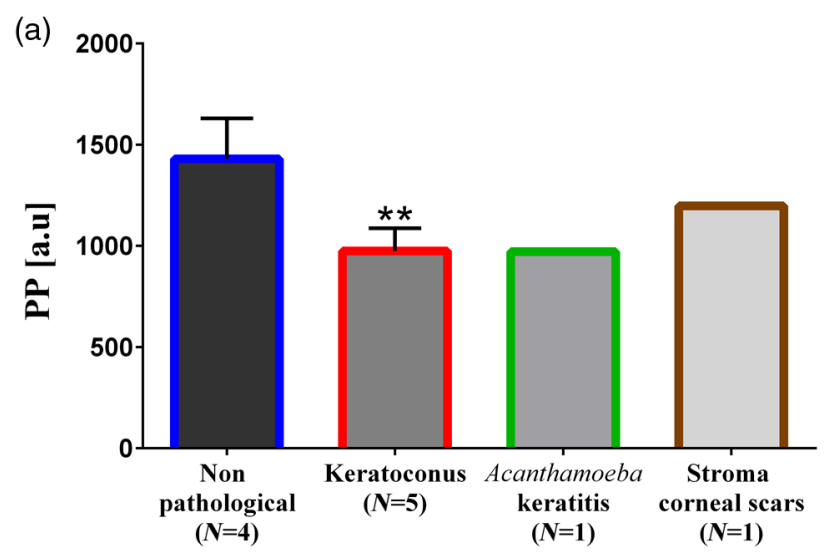

stroma at multiple depths for NP, AK, KC, and SCS corneas. Qualitative differences between all pathologies can be observed (Fig. 5). The FFT analysis of the SHG images was used to quantitatively assess alterations of organization of collagen fibers [Fig. 6(a)]. For KC, a significant decrease of PP was observed,

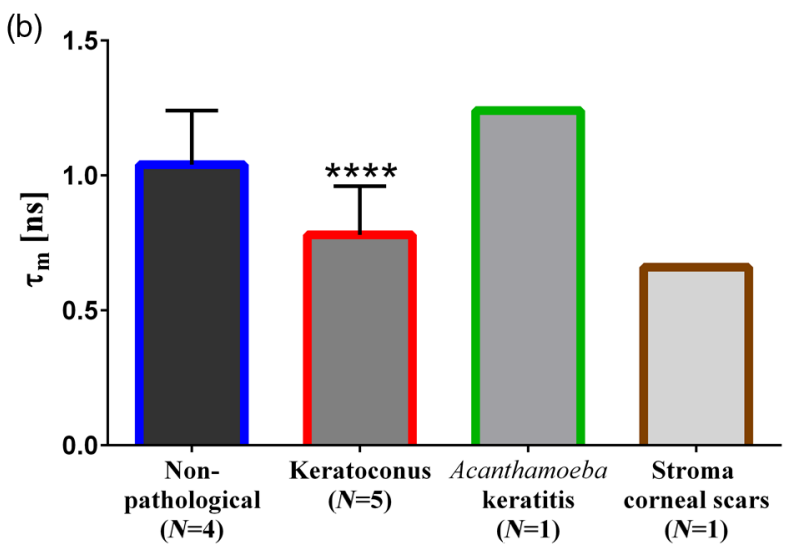

Fig. 6 (a) Degree of orientation of the stroma collagen fibers in nonpathological human corneas and corneas with AK, KC, and SCS assessed using the PP. (b) Mean stroma AF lifetimes of nonpathological and disease-affected corneas. Values represent mean \pm standard deviation. ${ }^{*}$ Statistically significant difference $(p<0.01)$; ${ }^{* \star \star}$ Statistically significant at $p<0.0001$. 
indicating a decrease in the orientation of the collagen fibers. A tendency for a decrease was also observed for AK and SCS samples [Fig. 6(a)].

Along with changes of the collagen stroma organization, significant differences were observed for the AF lifetime $\tau_{m}$. It was significantly decreased in KC corneas when compared with NP corneas [Fig. 6(b)]. SCS corneas also show a tendency for a decrease, while AK shows a tendency for an increase in the stroma $\tau_{m}$ [Fig. 6(b)].

\section{Discussion}

The early diagnosis of corneal pathologies is an important precondition for a good prognosis. Until now, advances of cornea imaging enabled earlier and earlier detection. The development of confocal microscopy provided additional information to the clinical features of AK, particularly the in vivo visualization of amoeba cysts, and aided AK diagnosis. ${ }^{19}$ However, AK is still often misdiagnosed. ${ }^{5}$ Corneal topography greatly improved the detection of $\mathrm{KC}$; however, the results can occasionally be unclear. ${ }^{5}$ Moreover, the biological mechanisms of this disease are not fully understood. ${ }^{17}$ Other tools are required to understand them and provide earlier diagnosis. SCS can be formed following cornea injury, infection, or even after refractive surgery and leads to cornea opacification, which can be observed through slit lamp biomicroscopy. ${ }^{20}$ Instruments capable of detecting structural modifications prior to cornea opacification are needed.

NP human corneas as well as corneas diagnosed with $\mathrm{KC}$, $\mathrm{AK}$, and SCS were characterized with high-resolution and without external labels using TPI. We could characterize not only the specific morphological alterations induced by these pathologies but also alterations to the epithelial cells' metabolism as well as to the stroma $\mathrm{AF}$ and structural organization.

The effect of different pathologies on the epithelial cells' morphology was revealed using the cells' AF intensity (Fig. 2). Spectral analysis of the cornea epithelial cells' AF revealed $\mathrm{NAD}(\mathrm{P}) \mathrm{H}$ as the major sources of $\mathrm{AF}$. Other sources of $\mathrm{AF}$, including flavins, were negligible possibly due to the low concentration of this fluorophore in the cornea. ${ }^{24}$ Thus, a spectral detection range suitable for $\mathrm{NAD}(\mathrm{P}) \mathrm{H}$ detection was chosen. This metabolic cofactor, mainly distributed in the mitochondria and the cell cytoplasm, ${ }^{8}$ allows visualization of single cells and their morphological characteristics (Fig. 2). Furthermore, the tissue AF lifetime provides additional insight on the tissue alterations due to pathologies (Fig. 3).

In corneas diagnosed with $\mathrm{KC}$, an increase in the epithelial cell size accompanied by an elongation of the cells was observed (Fig. 2). This is consistent with the reported anatomic modifications induced by KC to the epithelial layer. ${ }^{17}$ The AF lifetime images also showed an increase in the cells' heterogeneity, and cells with different $\mathrm{NAD}(\mathrm{P}) \mathrm{H} \tau_{m}$ were visualized (Fig. 3). As anticipated, a decrease in the corneal epithelial thickness was also observed. , $17^{2}$

Interestingly, a unique cellular membrane AF was seen in the case of AK cornea (Fig. 2). The cellular membrane AF exhibited a longer fluorescence lifetime than the remaining cellular structures, which provides an additional means of discrimination (Fig. 3). This alteration observed in the epithelial layer is likely related to the gradual infiltration of pathogenic organisms into the cornea. AK results from an infestation by the free-living amoeba, which starts with the attachment of the amoeba to the corneal epithelial cells and leads to degradation of the epithelial layer and stroma and infiltration of deeper layers of the cornea. ${ }^{5,18}$ A decrease in the corneal epithelial thickness along with regions where basal cells were absent was also observed, possibly due to cell degradation promoted by the local triple therapy consisting of propamidine isetionate, polyhexanide, and antibiotic eye drops (Fig. 2, red arrows). However, further studies with more AK samples are needed to understand better the AF modifications.

In the SCS cornea, a slight increase in the epithelial cell sizes was observed, particularly for basal cells. A possible explanation is that the epithelial cell layer can smooth the anterior surface of the eye by augmented proliferation and fill gaps induced by the scarring process. ${ }^{25}$ Furthermore, autofluorescent spots, possibly due to deposition of debris, were observed in the epithelial cells (Fig. 2). These debris AF had a short mean lifetime and homogenous distribution, which can be used to better discriminate between debris and cellular components (Fig. 3).

Based on the AF lifetime of the metabolic cofactor NAD(P) $\mathrm{H}$, the metabolic activity of epithelial cells was assessed for NP as well as for KC, AK, and SCS corneas. Significant differences of the NAD(P)H $a_{1} / a_{2}$ ratios were observed between NP and $\mathrm{KC}$ corneas (Fig. 4). In KC, NAD(P)H $a_{1} / a_{2}$ ratios of epithelial cells were significantly higher than in NP. Since oxidation and reduction are catalyzed by enzymes (proteins), the ratio between free $\left(a_{1}\right)$ and protein-bound $\left(a_{2}\right)$ components of $\mathrm{NAD}(\mathrm{P}) \mathrm{H}$ provides an indirect measure of the oxidation-reduction ratio and, therefore, of the cell metabolism. An increase in the NAD $(\mathrm{P}) \mathrm{H}$ $a_{1} / a_{2}$ ratio indicates an increase of free $\mathrm{NAD}(\mathrm{P}) \mathrm{H}$ and/or a decrease of the protein-bound component. This has been previously associated with a decrease in the metabolic activity of cells. ${ }^{26}$ Although an increase in the $\mathrm{NAD}(\mathrm{P}) \mathrm{H} a_{1} / a_{2}$ ratio was also observed for AK and SCS, statistical significance could not be assessed due to the low number of samples with these diseases. This indicates that pathological conditions may induce alterations to the metabolic activity of epithelial cells.

Changes of the stroma AF and SHG signals resulting from pathological conditions were evaluated. Alterations of the structural organization of the corneal stroma due to $\mathrm{AK}, \mathrm{KC}$, and SCS were observed based on SHG imaging [Figs. 5 and 6 (a)]. The orientation of the collagen fibers was analyzed by an FFT-based method. In KC corneas, a significant decrease in the degree of the collagen fiber orientation was observed [Fig. 6(a)]. Corneal ectatic disorders such as $\mathrm{KC}$ are known to induce alterations to the cornea stroma. For $\mathrm{KC}$ corneas, a decrease in the stroma thickness and a significant decrease in the number of collagen lamellae at the cone have been reported. ${ }^{5,17}$ Hence, alterations of the collagen fibers organization could be anticipated [Fig. 6(a)]. SHG imaging has already shown alterations of the collagen lamellae distribution patterns induced by $\mathrm{KC}^{27}$ and their levels of interweaving, ${ }^{28}$ as well as their orientations. ${ }^{29,30}$

The SHG images of AK corneas show visible alterations when compared with NP corneas (Fig. 5). Moreover, a decrease of PP was observed for this pathology, indicating a tendency for a decrease in the collagen fibers organization [Fig. 6(a)]. In AK, the contaminating organisms degrade the corneal stroma to facilitate their penetration into deeper layers. ${ }^{5,17}$ Therefore, a rearrangement of the collagen fibers with a lower degree of organization could be anticipated. The feasibility of AF and SHG imaging to assess morphological alterations induced by bacterial and fungal infections of the corneas has also been reported..$^{31,32}$ 
SCS are formed during abnormal wound healing. When the cornea is injured, the keratocytes are stimulated and migrate to the affected area. These cells are usually in a quiescent state and maintain the corneal stroma through the production of extracellular matrix components including collagen. ${ }^{5}$ However, during wound healing, keratocytes may differentiate into myofibroblasts, which are themselves more opaque than keratocytes and produce high quantities of disorganized extracellular matrix components finally leading to cornea opacification. ${ }^{20}$ Therefore, alterations of the organization of collagen fibers were expected [Fig. 6(a)]. The feasibility of TPI to monitor the scar tissue formation during wound healing has been demonstrated. ${ }^{33}$

In addition to SHG, the collagen and keratocytes in the corneal stroma generate AF signals. In this study, differences of the stroma mean AF lifetimes were observed between NP and pathological corneas as well as between different pathologies [Fig. 6(b)]. This shows that the stroma AF can provide additional information on this layer and help to better discriminate between NP and pathological as well as between different pathologies.

\section{Conclusion}

Using TPI, we characterized ex vivo human corneas based on the morphology and metabolism of the epithelial cells as well as on the stroma $\mathrm{AF}$ and the structural arrangement of its collagen fibers. Based on these characteristics, which are, except for the cell morphology, inaccessible with current clinical devices, non-pathological and pathological corneas could be discriminated. For KC corneas, the characteristic morphological alterations were observed. We demonstrated that TPI can provide additional information and may eventually enable a better evaluation of this corneal pathology. Although alterations of the morphology of cells as well as indications of altered cell metabolism, corneal stroma AF lifetime, and structural organization were observed for AK and SCS corneas, the analysis of further samples is necessary to be able to draw definite conclusions.

The results indicate that TPI can provide additional information on the cornea to improve the diagnosis of corneal pathologies as well as to understand the pathophysiology of these conditions. To the best of our knowledge, this was the first time that epithelial cells and stromal AF lifetime, combined with stroma SHG imaging, were used to evaluate alterations induced by these pathologies. In the future, we intent to use mobile TPI systems in a clinical environment to evaluate human cornea samples immediately after keratoplasty and set new diagnostic markers and improve and anticipate the diagnosis of the corneal diseases.

\section{Disclosures}

Karsten König is the CEO of JenLab GmbH.

\section{Acknowledgments}

The authors would like to thank Andrea Kaiser for logistic support. We acknowledge the support by the European Union's Horizon 2020 research and innovation program under Grant Agreement No. 726666 (LASER-HISTO).

\section{References}

1. J. P. Whitcher, M. Srinivasan, and M. P. Upadhyay, "Corneal blindness: a global perspective," Bull World Health Organ. 79(3), 214-221 (2001).
2. S. Agarwal et al., Textbook of Ophthalmology, Lippincott Williams \& Wilkins, Philadelphia (2002).

3. D. M. Maurice, "Cellular membrane activity in the corneal endothelium of the intact eye," Experientia 24, 1094-1095 (1968).

4. M. Petran et al., "Tandem-scanning reflected-light microscope," J. Opt. Soc. Am. 58(5), 661-664 (1968).

5. J. H. Krachmer, M. J. Mannis, and E. J. Holland, Cornea, 3rd ed., Mosby/Elsevier, St. Louis, Missouri (2011).

6. E. M. Messmer, "Confocal microscopy: when is it helpful to diagnose corneal and conjunctival disease?" Expert Rev. Ophthalmol. 3(2), 177192 (2008).

7. M. C. Skala et al., "In vivo multiphoton microscopy of NADH and FAD redox states, fluorescence lifetimes, and cellular morphology in precancerous epithelia," Proc. Natl. Acad. Sci. U. S. A. 104(49), 19494-19499 (2007).

8. I. Georgakoudi and K. P. Quinn, "Optical imaging using endogenous contrast to assess metabolic state," Annu. Rev. Biomed. Eng. 14, 351-367 (2012).

9. L. Tian et al., "Microscopic second-harmonic generation emission direction in fibrillous collagen type I by quasi-phase-matching theory," J. Appl. Phys. 108(5), 54701-54709 (2010).

10. D. W. Piston, B. R. Masters, and W. W. Webb, “Three-dimensionally resolved $\mathrm{NAD}(\mathrm{P}) \mathrm{H}$ cellular metabolic redox imaging of the in situ cornea with two-photon excitation laser scanning microscopy," J. Microsc. 178(1), 20-27 (1995).

11. S. W. Teng et al., "Multiphoton autofluorescence and second-harmonic generation imaging of the ex vivo porcine eye," Invest. Ophthalmol. Visual Sci. 47(3), 1216-1224 (2006).

12. A. Batista et al., "Two-photon spectral fluorescence lifetime and second-harmonic generation imaging of the porcine cornea with a 12 femtosecond laser microscope," J. Biomed. Opt. 21(3), 036002 (2016).

13. K. König, "High-resolution multiphoton imaging and nanosurgery of the cornea using femtosecond laser pulses," in Lasers in Ophthalmology: Basic, Diagnostic, and Surgical Aspects: a Review, F. Fankhauser and S. Kwasniewska, Eds., Kugler Publications, Amsterdam (2003).

14. F. Aptel et al., "Multimodal nonlinear imaging of the human cornea," Invest. Ophthalmol. Visual Sci. 51(5), 2459-2465 (2010).

15. A. Batista et al., "Assessment of human corneas prior to transplantation using high-resolution two-photon imaging," Invest. Opthalmol. Visual Sci. 59(1), 176 (2018).

16. K. König et al., "First in vivo animal studies on intraocular nanosurgery and multiphoton tomography with low-energy $80-\mathrm{MHz}$ near-infrared femtosecond laser pulses," Proc. SPIE 5314, 262-269 (2004).

17. M. L. Khaled et al., "Molecular and histopathological changes associated with keratoconus," Biomed Res. Int. 2017, 7803029 (2017).

18. D. W. Clarke and J. Y. Niederkorn, "The pathophysiology of Acanthamoeba keratitis," Trends Parasitol. 23(4), 175-180 (2006).

19. B. Clarke et al., "Advances in the diagnosis and treatment of Acanthamoeba keratitis," J. Ophthalmol. 2012, 484892 (2012).

20. A. A. M. Torricelli et al., "The corneal fibrosis response to epithelialstromal injury," Exp. Eye Res. 142(6), 110-118 (2016).

21. P. Elisabeth, B. Hilde, and C. Ilse, "Eye bank issues: II. Preservation techniques: warm versus cold storage," Int. Ophthalmol. 28(3), 155163 (2008).

22. A. Batista et al., "Characterization of porcine eyes based on autofluorescence lifetime imaging," Proc. SPIE 9329, 93290E (2015).

23. Y. Mega et al., "Quantification of lamellar orientation in corneal collagen using second harmonic generation images," Opt. Lett. 37(16), 3312-3314 (2012).

24. D. W. Batey and C. D. Eckhert, "Analysis of flavins in ocular tissues of the rabbit," Invest. Ophthalmol. Visual Sci. 32(7), 1981-1985 (1991).

25. L. Lu, P. S. Reinach, and W. W. Kao, "Corneal epithelial wound healing," Exp. Biol. Med. 226(7), 653-664 (2001).

26. K. König et al., "Applications of multiphoton tomographs and femtosecond laser nanoprocessing microscopes in drug delivery research," Adv. Drug Delivery Rev. 63(4-5), 388-404 (2011).

27. H. Y. Tan et al., "Multiphoton fluorescence and second harmonic generation imaging of the structural alterations in keratoconus ex vivo," Invest. Ophthalmol. Visual Sci. 47(12), 5251-5259 (2006).

28. N. Morishige et al., "Second-harmonic imaging microscopy of normal human and keratoconus cornea," Invest. Ophthalmol. Visual Sci. 48(3), 1087-1094 (2007). 
29. N. Morishige et al., "Quantitative analysis of collagen lamellae in the normal and keratoconic human cornea by second harmonic generation imaging microscopy," Invest. Ophthalmol. Visual Sci. 55(12), 83778385 (2014).

30. R. Mercatelli et al., "Three-dimensional mapping of the orientation of collagen corneal lamellae in healthy and keratoconic human corneas using SHG microscopy," J. Biophotonics 83(1), 75-83 (2016).

31. H.-Y. Tan et al., "Multiphoton fluorescence and second harmonic generation microscopy for imaging infectious keratitis," J. Biomed. Opt. 12(2), 024013 (2007).

32. D. M. Robertson et al., "Second harmonic generation imaging of corneal stroma after infection by Pseudomonas aeruginosa," Sci. Rep. 7, 46116 (2017).

33. S. W. Teng et al., "Multiphoton fluorescence and second-harmonic-generation microscopy for imaging structural alterations in corneal scar tissue in penetrating full-thickness wound," Arch. Ophthalmol. 125(7), 977-978 (2007).

Ana Batista received her master's degree in biomedical engineering in 2010 at the Faculty of Sciences and Technology, University of Coimbra. In April 2016, she enrolled in the PhD program of the University of Saarland. Her current research activities aim to study the morphology and metabolism of corneal tissues using multiphoton microscopy. Her work already resulted in participation in several scientific meetings and scientific paper publications.

Hans Georg Breunig is a physicist and senior research scientist at JenLab $\mathrm{GmbH}$. He holds a master's degree from the University of Maryland, College Park, USA, and received his PhD from the University of Bremen, Germany. He has worked for more than fifteen years on applications of femtosecond lasers in different research fields and is the author of more than 40 journal papers.

Aisada König received her PhD from Saarland University, Faculty of Medicine, Homburg, Germany, and worked as a postdoctoral researcher at the University of California, San Diego (UCSD). She is the PI of the German Science Foundation funded project on optical reprogramming and embarked on habilitation in the field of femtosecond laser microscopy of stem cells at Saarland University.

Andreas Schindele earned his master's degree in mathematics in science and engineering at TU Munich in 2012. After gaining his doctorate about MRI image reconstruction and optimal control in 2016, he is now developing image analyzation software for JenLab $\mathrm{GmbH}$, Jena, Germany. He specializes in image processing and medical applications..

Tobias Hager studied human medicine at the CharitéUniversitästsmedizin in Berlin between 2001 and 2008. Since 2008, he is an ophthalmologist at the Department of Ophthalmology at Saarland University Medical Center, Germany. He completed his PhD in July 2008 and his medical speciality at the Department of Ophthalmology at Saarland University Medical, Germany in 2013.

Berthold Seitz is a full professor of ophthalmology and chairman of the Department of Ophthalmology, Saarland University Medical Center, Homburg/Saar, Germany, since March 2006. Since September 2001, he is a founder and secretary of Sektion Kornea in der DOG. Since August 2011, he is a member of the National Academy of Sciences Leopoldina (ML). From 2012 to 2013, he is a president of the German Ophthalmological Society (DOG). Since February 2016, he has been a director of the ICO Fellowship Program.

Karsten König studied physics in Rostock, wrote his PhD and habilitation thesis in Jena, and worked at the Institute for Laser Technologies in UIm, Beckman Laser Institute in Irvine, Institute for Molecular Biotechnology in Jena and University Jena. Currently, he is a full professor for biophotonics and laser technology at the Faculty of Physics and Mechatronics, Saarland University, Saarbrücken, Germany. He is a cofounder of the spin-off company JenLab $\mathrm{GmbH}$. He is a author of more than 200 publications and received a variety of awards such as the Fraunhofer Award Technik für den Menschen, the Kortum Motivationspreis, the Pascal Rol Award of the SPIE, the Feulgen Price and the Award for Applied Research of the State of Thuringia. 